\title{
Laminotomy/medial facet approach in the excision of thoracic disc herniation
}

\author{
Perry BLack, M.D. \\ Department of Neurosurgery, MCP Hahnemann University, Philadelphia, Pennsylvania
}

\begin{abstract}
Object. The author describes a technique of thoracic discectomy that has evolved from the posterolateral transfacet and the transpedicular approaches but that spares the pedicle and most of the facet joint.

Methods. This approach was used to remove a total of 11 discs (T6-12) in seven patients. The follow-up period ranged from 8 months to 3 years. In four patients with axial and/or girdle pain significant improvement was demonstrated. The paraparesis in one patient with myelopathy improved postoperatively; that in another patient improved but recurred 8 months postoperatively. In one patient who experienced preoperative leg weakness, the weakness was slightly increased postoperatively, but this sequela was only transient. There were no other complications, and there were no deaths.

Conclusions. This technique appears safe and effective. It can be adapted to the conventional laminectomy known to spine surgeons and requires no specialized instruments. Further trials appear warranted.
\end{abstract}

KEY WORDS • thoracic spine • herniated disc - laminotomy • facet

Excision of a herniated thoracic disc continues to pose a surgical challenge. Historically, attempts at disc removal via a laminectomy approach have generally been associated with poor results. ${ }^{3,9}$ Neurosurgeons have come to regard the spinal cord as a structure that tolerates neither retraction nor contusion. In response to the intolerance of the spinal cord to manipulation, a range of surgical trajectories has been developed over recent decades in an effort to decompress the spinal cord and adjacent nerve roots safely. Disc excision via the transthoracic approach, first described by Perot and Munro ${ }^{6}$ and by Ransohoff, et al., ${ }^{7}$ proved to be an effective but formidable technique. Extracavitary approaches were subsequently introduced. Among these were the lateral extracavitary ${ }^{4}$ and the costotransversectomy approaches. ${ }^{1}$ Although these extracavitary approaches were also found to be effective, they are moderately extensive surgical undertakings and entail considerable bone removal.

More limited posterolateral approaches were being introduced at the same time that microsurgical techniques were first described. The transpedicular procedure, introduced by Patterson and Arbit, ${ }^{5}$ was followed by the transfacet pedicle-sparing approach described by Stillerman, et al. ${ }^{8}$ A thoracic endoscopic technique has also been introduced. ${ }^{2}$ In this paper an alternative posterolateral technique is presented that has evolved from the posterolateral transfacet and the transpedicular approaches but that spares the pedicle and most of the facet joint.

\section{CLINICAL MATERIAL AND METHODS}

\section{Patient Population}

The approach was used to remove a total of 11 discs in seven patients. The herniated discs were all located in the lower half of the thoracic spine, from T-6 to T-12. One of the patients underwent removal of three herniated discs, and two patients each underwent removal of two discs. In the remaining four patients one disc was excised; one of these patients underwent a bilateral laminectomy to correct central spinal canal stenosis, in addition to removal of the herniated disc. Four patients suffered axial and/or radicular (girdle) pain, two patients (one with myelopathy), suffered paraparesis, and one patient with myelopathy experienced leg weakness.

\section{Preoperative Preparation}

Preoperative Preparation for Intraoperative Localization of Disc Level. To facilitate localization of the correct disc level at the time of surgery, a preoperative chest x-ray film study is obtained to determine the lowest rib and the number of the corresponding vertebral body. The x-ray film is compared with the localization of the involved disc as demonstrated on magnetic resonance imaging. Prior to making the operative incision, anteroposterior fluoroscopy (or a plain radiography) is used to enable the surgeon to count the thoracic disc spaces starting from the lowest rib. As suggested by Kumar and Dunsker, ${ }^{3}$ counting of the disc spaces is aided by placing multiple metal markers on the skin overlying the spine.

Electrophysiological Monitoring. Electrodes are applied to the patient's limbs and scalp for continuous intraoperative spinal cord monitoring of somatosensory evoked potentials as well as transcranial motor potentials. Inhalational anasthetic agents are not used in conjunction with electrophysiological monitoring because they interfere with electrical conduction. 


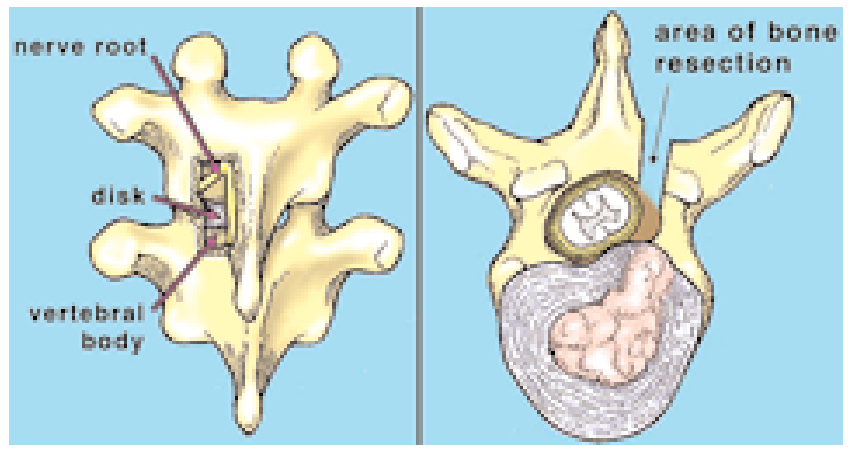

Fig. 1. Drawings depicting the area of bone resection required to expose the disc and the lateral edge of the thecal sac. A unilateral interlaminar laminotomy involving the superior and inferior laminar arches is conducted, as well as of the medial 2 to $3 \mathrm{~mm}$ of the facet joint. Left: Posterior view. Note the exiting nerve root superior to the disc. Right: Axial view.

\section{Surgical Technique}

Surgical Exposure. A midline incision, approximately $5 \mathrm{~cm}$ in length, is centered over the involved disc. When the spinous processes are reached, a spinal needle is inserted in the interspinous ligament as a marker for repeated fluoroscopic imaging to check the disc level. Unilateral subperiosteal dissection of the muscle is performed on the side of the more prominent lesion. An interlaminar laminotomy is conducted using a drill and/or a $45^{\circ}$ angled Kerrison-type rongeur. The exposed field includes the lateral edge of the thecal sac and the medial 2 to $3 \mathrm{~mm}$ of the facet joint (Fig. 1). This provides exposure of approximately $5 \mathrm{~mm}$ of the lateral portion of the disc, which is sufficient to undertake the discectomy (see Disc Excision). If necessary, an additional 1 to $2 \mathrm{~mm}$ of the facet joint may be removed.

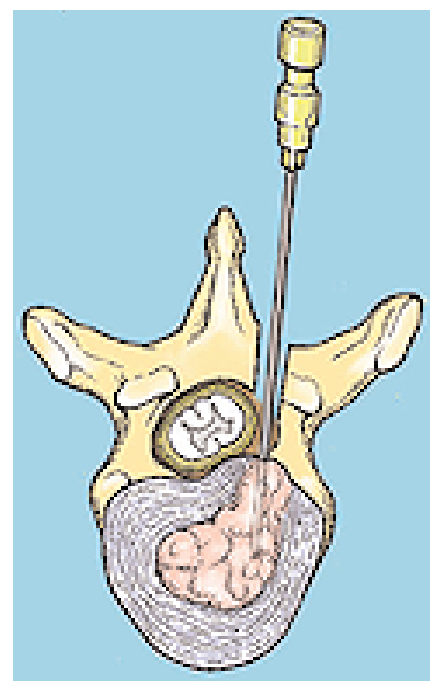

Fig. 2. Drawing illustrating a No. 20 spinal needle used to probe the posterior aspect of the vertebral body to help locate the disc. Final confirmation of the correct disc level is then made fluoroscopically or by plain radiography.

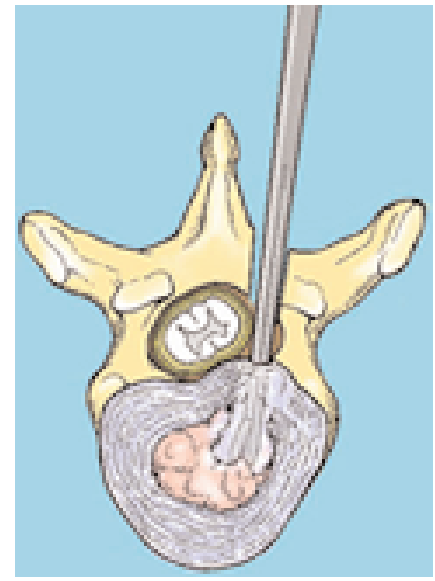

Fig. 3. Drawing showing micropituitary rongeurs, which are used to remove disc fragments to create a small cavity in the disc space.

The exposure enables insertion and manipulation of the instruments lateral to and beneath the dura mater at varying angles. This facilitates removal of disc material and osteophytic spurs in the midline and on the contralateral side of the disc. Exposure of the dura confers a measure of safety to the procedure by keeping the dura constantly in view as instruments are manipulated in the disc space and at the endplate margins.

The surface of the disc is generally not readily differentiated from the soft tissue covering the posterior longitudinal ligament over the posterior aspect of the vertebral body. A spinal needle is used to probe the posterior portion of the vertebral body until the softer disc space is located. Final confirmation of the correct disc level is made fluoroscopically, or by plain radiography while the spinal needle is in place (Fig. 2).

Disc Excision. Using the microscope or loupes with headlight illumination, a rectangular incision is made in the disc capsule, and disc fragments are removed using micropituitary rongeurs (Fig. 3). After a small cavity has been created in the disc space, an Epstein reverse cup curette is used to resect the superior and inferior disc endplates (Fig. 4). To avoid spinal cord injury, fragments of disc material are displaced downward away from the ventral dura and into the disc space, from which the fragments are then removed using straight, up- and downbiting micropituitary rongeurs.

Excision of Osteophytic Spurs. Once the disc material is removed, the osteophytic spurs, which project into the spinal canal from the margins of the endplates, are resected. Beginning laterally and moving medially, these spurs are dislodged and then displaced downward with the Epstein reverse cup curettes into the disc space (Fig. 5). The fragments are then extracted from the disc space with micropituitary rongeurs.

\section{RESULTS}

In four patients with axial and/or radicular (girdle) pain, significant improvement was observed. The preoperative paraparesis in one patient with myelopathy improved, and 


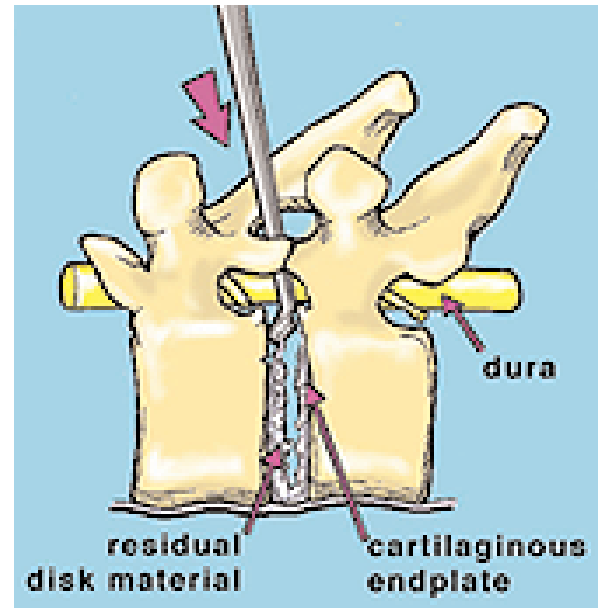

Fig. 4. A small Epstein reverse cup curette is used to resect the superior and inferior cartilaginous endplates, as well as residual disc material. Fragments of cartilage and disc are displaced downward into the cavity, away from the ventral dura mater. The fragments are then removed from the disc space by using straight, upand downbiting micropituitary rongeurs.

that in another patient improved but recurred 8 months postoperatively. In one patient with myelopathy who experienced preoperative leg weakness, the weakness was transiently slightly increased postoperatively. There were no other complications and no deaths.

In all cases after resection of the disc material and the osteophytic spurs, the spine appeared to be stable. Patients were ambulatory on the day following surgery without need for external immobilization with a brace. The follow-up period ranged from 8 months to 3 years.

\section{CONCLUSIONS}

The preliminary clinical experience reported here would suggest that the technique is safe and effective. The approach is comparable with the other posterolateral procedures - transpedicular and transfacet-with respect to the surgical trajectory and the relatively small amount of bone removed. One advantage of the laminotomy/medial facet approach described here is that the pedicle and most of the facet joint are preserved. In other posterolateral techniques, the resection of disc and osteophytic spurs in the midline and contralaterally is performed blindly in that the instruments are inserted beneath the dura. In the present technique, this lack of visualization is compensated for by the use of an Epstein reverse cup curette, which displaces the disc material and spurs downward, always away from the dural sac and into the disc space. The dural sac and spinal cord are never retracted. Electrophysiological monitoring of spinal cord function provides additional safety. The operative exposure may not be adequate to open the dura for the removal of an intradural disc fragment, which occurs only rarely. The exposure is similarly insufficient to perform an interbody fusion if this should be necessary due to preexisting spinal instability.

This approach can be readily adopted by spine surgeons who are comfortable with the laminectomy approach, and no special instruments are needed. Based on this preliminary clinical experience, further trials appear warranted.

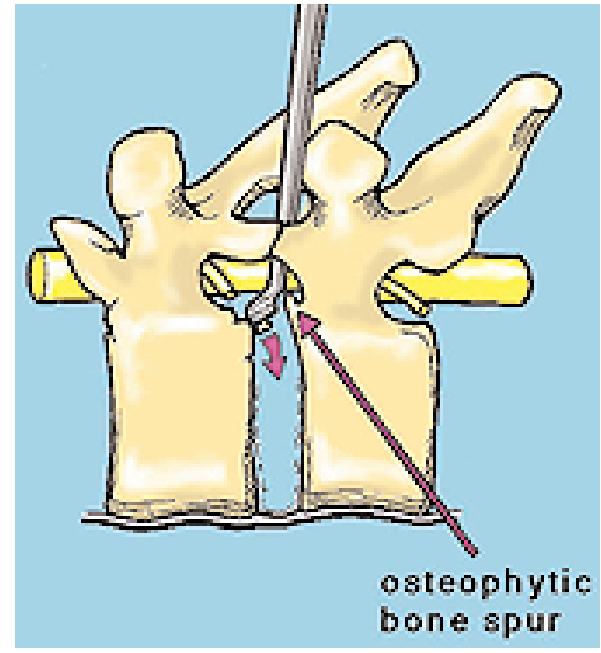

Fig. 5. The final step in the procedure is the resection of osteophytic spurs projecting into the spinal canal from the margins of the endplates. The spurs are dislodged with the Epstein reverse cup curette and displaced downward, away from the ventral dura mater. The bone fragments are then extracted from the disc space by using the micropituitary rongeurs.

\section{Acknowledgment}

The illustrations in this paper were prepared by Lynn Reynolds, A.M.I., ARS Medica, Doylestown, Pennsylvania.

\section{References}

1. Hulme A: The surgical approach to thoracic intervertebral disc protrusions. J Neurol Neurosurg Psychiatry 23:133-137, 1960

2. Krasna M, Mack M: Atlas of Thorascopic Surgery. St. Louis: Quality Medical, 1994, pp 206-211

3. Kumar R, Dunsker SB: Surgical management of thoracic disc herniations, in Schmidek HH, Sweet WH (eds): Operative Neurosurgical Techniques, ed 3. Philadelphia: WB Saunders, 1995, pp 1895-1904

4. Maiman DJ, Larson SJ, Luck E, et al: Lateral extracavitary approach to the spine for thoracic disc herniation: report of 23 cases. Neurosurgery 14:178-182, 1984

5. Patterson RH Jr, Arbit E: A surgical approach through the pedicle to protruded thoracic discs. J Neurosurg 48:768-772, 1978

6. Perot PL Jr, Munro DD: Transthoracic removal of midline disc protrusions causing spinal cord compression. J Neurosurg 31: 452-458, 1969

7. Ransohoff J, Spencer F, Siew F, et al: Transthoracic removal of thoracic disc. Report of three cases. J Neurosurg 31:459-461, 1969

8. Stillerman CB, Chen TC, Day JD, et al: The transfacet pediclesparing approach for thoracic disc removal: cadaveric morphometric analysis and preliminary clinical experience. J Neurosurg 83:971-976, 1995

9. Stillerman CB, Weiss MH: Surgical management of thoracic disc herniation and spondylosis, in Menezes AH, Sonntag VKH (eds): Principles of Spinal Surgery. New York: McGraw-Hill, 1996, pp 581-601

Manuscript received August 15, 2000.

Accepted in final form September 8, 2000

Address reprint requests to: Perry Black, M.D., Department of Neurosurgery, MCP Hahnemann University, Mail Stop 407, 245 North 15th Street, Philadelphia, Pennsylvania 19102. 